\title{
Evaluation of ground state entanglement in spin systems with the random phase approximation
}

\author{
J.M. Matera, R. Rossignoli, N. Canosa \\ Departamento de Fúsica-IFLP, Universidad Nacional de La Plata, C.C. 67, La Plata (1900) Argentina
}

(Dated: July 3, 2018)

\begin{abstract}
We discuss a general treatment based on the mean field plus random phase approximation (RPA) for the evaluation of subsystem entropies and negativities in ground states of spin systems. The approach leads to a tractable general method, becoming straightforward in translationally invariant arrays. The method is examined in arrays of arbitrary spin with $X Y Z$ couplings of general range in a uniform transverse field, where the RPA around both the normal and parity breaking mean field state, together with parity restoration effects, are discussed in detail. In the case of a uniformly connected $X Y Z$ array of arbitrary size, the method is shown to provide simple analytic expressions for the entanglement entropy of any global bipartition, as well as for the negativity between any two subsystems, which become exact for large spin. The limit case of a spin $s$ pair is also discussed.

PACS numbers: 03.67.Mn, 03.65.Ud, 75.10.Jm
\end{abstract}

\section{INTRODUCTION}

The study of entanglement constitutes one of the most active and challenging research areas, being of central interest in the fields of quantum information [1] and manybody physics [2]. The concept of entanglement has provided a new perspective for analyzing quantum correlations and quantum critical phenomena in many particle systems, leading to fundamental results and new insights in the field [2 5]. Nonetheless, the evaluation of entanglement in general strongly interacting many-body systems remains a difficult task, particularly in systems with long range interactions, high connectivity and large dimensionality, where usual treatments such as Quantum MonteCarlo [6], DMRG [7] or matrix product states [8] become more involved or difficult to implement. In previous works 9, 10] we have applied a general mean field plus RPA treatment to the evaluation of pairwise entanglement (i.e., that between two elementary components) in spin systems at zero and finite temperature. The approach was able to capture the main features of the entanglement between two spins in arrays with $X Y$ and $X Y Z$ couplings of different ranges, including the prediction of full range pairwise entanglement in the vicinity of the factorizing field 10 12]. The accuracy of the approach was shown to increase with the interaction range or connectivity.

The aim of the present work is to examine the capability of the previous method for predicting, in the ground state of spin systems, the entanglement properties of general subsystems. We will focus on the entanglement entropy of arbitrary bipartitions of the whole system, as well as on the negativity between any two subsystems, not necessarily complementary, where the rest of the spins play the role of an environment and entanglement can no longer be measured through the subsystem entropy. Other measures, like the negativity (an entanglement monotone computable for general mixed states [13, 14]) have to be employed. This type of entanglement has recently received special attention [15 17] since its behavior can differ from that of global bipartitions. We will show that the present approximation provides a general tractable scheme for evaluating these quantities, becoming analytic in translationally invariant systems.

In Section II we present the general RPA formalism, describing the RPA spin state, the associated bosonic estimation of subsystem entropies and negativities, the implementation in translationally invariant systems and the application to a general spin $s$ array with $X Y Z$ couplings of arbitrary range in a transverse magnetic field. Symmetry restoration effects in the case of parity-breaking mean fields are also discussed. As illustration, we derive in sec. III results for a spin $s$ pair and for a fully connected finite spin $s$ array, where RPA is able to provide simple full analytic expressions for subsystem entropies and negativities, which represent the exact large spin limit at any fixed size. Appendix A discusses the equivalence between the spin and the bosonic RPA treatments, whereas appendix B contains details of the analytic results of sec. III. Conclusions are drawn in IV.

\section{FORMALISM}

\section{A. RPA for spin systems at $T=0$}

We will consider a general finite system of spins $\boldsymbol{s}_{i}=$ $\left(s_{i x}, s_{i y}, s_{i z}\right)$, connected through general quadratic couplings and immersed in a magnetic field, not necessarily uniform. The corresponding Hamiltonian is

$$
H=\sum_{i, \mu} B^{i \mu} s_{i \mu}-\frac{1}{2} \sum_{i \neq j, \mu, \nu} J^{i \mu j \nu} s_{i \mu} s_{j \nu},
$$

where $\mu=x, y, z$ and $B^{i \mu}$ are the field components at site $i$. Ising, $X Y, X Y Z\left(J^{i \mu j \nu}=\delta^{\mu \nu} J_{\mu}^{i j}\right)$ as well as Dzyaloshinskii-Moriya $\left(J^{i \mu, j \nu}=-J^{i \nu, j \mu}\right)$ couplings of arbitrary range are particular cases of Eq. (1).

The first step in the RPA [18] is to determine the mean 
field ground state, i.e., the separable state

$$
|0\rangle \equiv \otimes_{i=1}^{n}\left|0_{i}\right\rangle=\left|0_{1} \ldots 0_{n}\right\rangle
$$

with the lowest energy $\langle H\rangle_{0}=\langle 0|H| 0\rangle$, given by

$$
\langle H\rangle_{0}=\sum_{i, \mu} B^{i \mu}\left\langle s_{i \mu}\right\rangle_{0}-\frac{1}{2} \sum_{i \neq j, \mu, \nu} J^{i \mu j \nu}\left\langle s_{i \mu}\right\rangle_{0}\left\langle s_{j \nu}\right\rangle_{0}
$$

where $\left\langle\boldsymbol{s}_{i}\right\rangle_{0}=\left\langle 0_{i}\left|\boldsymbol{s}_{i}\right| 0_{i}\right\rangle$. Each local state $\left|0_{i}\right\rangle$ can be determined self-consistently as the lowest eigenstate of the local mean field Hamiltonian

$$
h_{i}=\sum_{\mu} \frac{\partial\langle H\rangle_{0}}{\partial\left\langle s_{i \mu}\right\rangle_{0}} s_{i \mu}=\boldsymbol{\lambda}^{i} \cdot \boldsymbol{s}_{i}
$$

being then the state with maximum spin $s_{i}$ directed along $-\boldsymbol{\lambda}^{i}$ (a local coherent state). This leads to the selfconsistent equations

$$
\lambda^{i \mu}=B^{i \mu}-\sum_{j \neq i, \nu} J^{i \mu j \nu}\left\langle s_{j \nu}\right\rangle_{0},\left\langle\boldsymbol{s}_{i}\right\rangle_{0}=-s_{i} \boldsymbol{\lambda}^{i} / \lambda^{i},
$$

where $\lambda^{i}=\left|\lambda^{i}\right|$. Eq. (4) can be solved iteratively starting from an initial guess for $\left|0_{i}\right\rangle$ or $\boldsymbol{\lambda}_{i}$, although other procedures (like the gradient method) can be employed. Eq. (22) becomes then $\langle H\rangle_{0}=\frac{1}{2} \sum_{i}\left(\boldsymbol{\lambda}^{i}+\boldsymbol{B}^{i}\right) \cdot\left\langle\boldsymbol{s}_{i}\right\rangle_{0}$.

Since the form (10) is valid for any choice of the local axes, it is now convenient to choose $z_{i}$ along $\boldsymbol{\lambda}^{i}$, such that $\left\langle s_{i \mu}\right\rangle_{0}=-s_{i} \delta_{\mu z}$ and $\lambda^{i \mu}=\lambda^{i} \delta^{\mu z}$, with $\lambda^{i}>0$. The second step in the RPA is the approximate bosonization

$$
s_{i+} \rightarrow \sqrt{2 s_{i}} b_{i}^{\dagger}, \quad s_{i-} \rightarrow \sqrt{2 s_{i}} b_{i}, \quad s_{i z} \rightarrow-s_{i}+b_{i}^{\dagger} b_{i},
$$

where $s_{i \pm}=s_{i x} \pm i s_{i y}$ and $b_{i}, b_{i}^{\dagger}$ are considered standard boson operators $\left(\left[b_{i}, b_{j}^{\dagger}\right]=\delta_{i j},\left[b_{i}, b_{j}\right]=\left[b_{i}^{\dagger}, b_{j}^{\dagger}\right]=0\right)$, with $|0\rangle \rightarrow\left|0_{b}\right\rangle$ their vacuum. This bosonization is in agreement with that implied by the path integral formalism of [9, 10] for $T \rightarrow 0$, and preserves two of the exact spin commutators exactly $\left(\left[s_{i}^{z}, s_{j}^{ \pm}\right]= \pm \delta_{i j} s_{i}^{ \pm}\right)$, the remaining one preserved as vacuum average $\left(\left\langle\left[s_{i}^{-}, s_{j}^{+}\right]\right\rangle_{0}=2 s_{i} \delta_{i j}\right)$. It coincides with the Holstein-Primakoff and other exact bosonizations [18 21] up to zeroth order in $s_{i}^{-1}$.

The third step is to replace Eq. (5) in the original Hamiltonian (1), neglecting all cubic and quartic terms in $b_{i}, b_{i}^{\dagger}$. This leads to the quadratic boson Hamiltonian

$$
\begin{aligned}
H^{b} & =\langle H\rangle_{0}+\sum_{i} \lambda^{i} b_{i}^{\dagger} b_{i}-\sum_{i \neq j} \Delta_{+}^{i j} b_{i}^{\dagger} b_{j}+\frac{1}{2}\left(\Delta_{-}^{i j} b_{i}^{\dagger} b_{j}^{\dagger}+\text { h.c. }\right) \\
& =\langle H\rangle_{0}-\frac{1}{2} \sum_{i} \lambda^{i}+\frac{1}{2} \mathcal{Z}^{\dagger} \mathcal{H} \mathcal{Z} \\
\mathcal{Z} & =\left(\begin{array}{c}
b \\
b^{\dagger}
\end{array}\right), \mathcal{H}=\left(\begin{array}{c}
\Lambda-\Delta_{+}-\Delta_{-} \\
-\bar{\Delta}_{-}
\end{array}\right), \\
\Delta_{ \pm}^{i j} & =\frac{1}{2} \sqrt{s_{i} s_{j}}\left[J^{i x j x} \pm J^{i y j y}-i\left(J^{i y j x} \mp J^{i x j y}\right)\right]
\end{aligned}
$$

where $\mathcal{Z}^{\dagger}=\left(b^{\dagger}, b\right)$ and $\Lambda^{i j}=\lambda^{i} \delta^{i j}$. The choice of the mean field axes for the bosonization (5) ensures that no linear terms in $b_{i}, b_{i}^{\dagger}$ appear in $H^{b}$, reflecting the stability of the mean field state $|0\rangle$ with respect to one site excitations.

The last step is the diagonalization of the bosonic quadratic form (6), which is always possible when the hermitian matrix $\mathcal{H}$ in (77) is positive definite, i.e., when $|0\rangle$ is a stable vacuum [18]. $H^{b}$ can then be rewritten as

$$
H^{b}=\langle H\rangle_{0}+\sum_{\alpha} \omega^{\alpha} b_{\alpha}^{\prime \dagger} b_{\alpha}^{\prime}+\frac{1}{2}\left(\omega^{\alpha}-\lambda^{\alpha}\right),
$$

where $\lambda^{\alpha}$ stands for $\lambda^{i}$, $\omega^{\alpha}$ are the symplectic eigenvalues of $\mathcal{H}$, i.e., the positive eigenvalues of the matrix

$$
\mathcal{M H}=\left(\begin{array}{cc}
\Lambda-\Delta_{+} & -\Delta_{-} \\
\bar{\Delta}_{-} & -\Lambda+\bar{\Delta}_{+}
\end{array}\right), \quad \mathcal{M}=\left(\begin{array}{cc}
1 & 0 \\
0 & -1
\end{array}\right)
$$

whose eigenvalues come in pairs of opposite sign (and which is diagonalizable with real non-zero eigenvalues when $\mathcal{H}$ is positive definite), and $b^{\prime}{ }_{\alpha}, b^{\prime \dagger}{ }_{\alpha}^{\dagger}$ are "collective" boson operators related to the local ones by a Bogoliubov transformation $\mathcal{Z}=\mathcal{W} \mathcal{Z}^{\prime}$, i.e.,

$$
\left(\begin{array}{c}
b \\
b^{\dagger}
\end{array}\right)=\mathcal{W}\left(\begin{array}{c}
b^{\prime} \\
b^{\prime \dagger}
\end{array}\right), \mathcal{W}=\left(\begin{array}{cc}
U & V \\
\bar{V} & \bar{U}
\end{array}\right)
$$

with $\left(\frac{U}{V}\right)_{\alpha}$ and $\left(\frac{V}{U}\right)_{\alpha}$ the eigenvectors of $\mathcal{M H}$ associated with the eigenvalues $\omega_{\alpha}$ and $-\omega_{\alpha}$ respectively (such that $\mathcal{W}^{-1} \mathcal{M H W}=\mathcal{M} \Omega$, with $\left.\Omega_{\alpha \alpha^{\prime}}=\left|\omega_{\alpha}\right| \delta_{\alpha \alpha^{\prime}}\right)$. In order to preserve the boson commutation relations, which can be cast as $\mathcal{Z Z}^{\dagger}-\left[\left(\mathcal{Z}^{\dagger}\right)^{\operatorname{tr}} \mathcal{Z}^{\operatorname{tr}}\right]^{\operatorname{tr}}=\mathcal{M}, \mathcal{W}$ should satisfy

$$
\mathcal{W} \mathcal{M} \mathcal{W}^{\dagger}=\mathcal{M}
$$

which implies also $\mathcal{W}^{\dagger} \mathcal{M W}=\mathcal{M}$ and hence $\mathcal{W}^{\dagger} \mathcal{H} \mathcal{W}=$ $\Omega$. This entails $U^{\dagger} V-V^{\operatorname{tr}} \bar{U}=0, U^{\dagger} U-V^{\operatorname{tr}} \bar{V}=I$, which are the natural orthogonality relations fulfilled by the eigenvectors of (10) with normalization $\left({ }_{V}^{U}\right)_{\alpha}^{\dagger} \mathcal{M}\left(\frac{U}{V}\right)_{\alpha}=1$.

The RPA matrix (7) is of dimension $2 n \times 2 n$, with $n$ the number of spins. RPA involves then an exponential reduction in the dimension (from $(2 s+1)^{n}$ to $2 n$ for $n$ identical spins). Moreover, in a translationally invariant system (sec. IID), it can be further reduced to $n 2 \times 2$ matrices, becoming then fully analytic.

\section{B. The RPA ground state}

The vacuum of the new bosons $b^{\prime}\left(b_{\alpha}^{\prime}\left|0_{b}^{\prime}\right\rangle=0\right)$ is [18]

$$
\left|0_{b}^{\prime}\right\rangle=C_{b} \exp \left[\frac{1}{2} \sum_{i, j} Z^{i j} b_{i}^{\dagger} b_{j}^{\dagger}\right]\left|0_{b}\right\rangle, \quad Z=V \bar{U}^{-1},
$$

where $C_{b}=\left\langle 0_{b} \mid 0_{b}^{\prime}\right\rangle=\operatorname{Det}[\bar{U}]^{-1 / 2}$ is a normalization factor and $Z$ a symmetric matrix. The associated RPA spin state can then be defined as

$$
\left|0_{\mathrm{RPA}}\right\rangle=C_{s} \exp \left[\frac{1}{2} \sum_{i \neq j} \frac{Z^{i j}}{2 \sqrt{s_{i} s_{j}}} s_{i+} s_{j+}\right]|0\rangle .
$$


The expectation values generated by (14) will be close to those obtained with the mapping (5), coinciding exactly up to second order in $V$ (Appendix A). In contrast with $|0\rangle$, the state (14) is entangled (unless $V \neq 0$ ).

Let us note that for the quadratic Hamiltonian (1):

i) $\left|0_{\mathrm{RPA}}\right\rangle=|0\rangle$ if and only if $|0\rangle$ is an exact eigenstate of $H$, since $H^{b}$ contains the exact matrix elements connecting $|0\rangle$ with the rest of the Hilbert space:

$$
H|0\rangle=\langle H\rangle_{0}|0\rangle-\frac{1}{2} \sum_{i, j} \Delta_{-}^{i j}\left|1_{i} 1_{j}\right\rangle,
$$

where $\left|1_{i} 1_{j}\right\rangle=\frac{s_{i+} s_{j+}}{2 \sqrt{s_{i} s_{j}}}|0\rangle$ and we have used the mean field condition $\left\langle 1_{i}|H| 0\right\rangle=\left\langle 1_{i}\left|h_{i}\right| 0_{i}\right\rangle=0$ (Eqs. (3)-(4)). Thus, if $\left|0_{\mathrm{RPA}}\right\rangle=|0\rangle, Z=0$ and hence $V=0$ in $\mathcal{W}$, implying $\Delta_{-}=0 .|0\rangle$ is then an exact eigenstate by Eq. (15). Conversely, if $|0\rangle$ is an exact eigenstate, it is a solution of the mean field equations leading to $\Delta_{-}=0$, implying $\left|0_{\mathrm{RPA}}\right\rangle=|0\rangle$ (although $\Delta_{+}$may be non-zero and $\left.\omega^{\alpha} \neq \lambda^{\alpha}\right)$. In particular, when $H$ has an exactly separable ground state $|0\rangle$ (i.e., at the factorizing field $[12,22,23]),\left|0_{\mathrm{RPA}}\right\rangle=|0\rangle$.

ii) $\left|0_{\text {RPA }}\right\rangle$ is always exact for sufficiently strong fields $(|\boldsymbol{B}| \gg J)$. In this limit $|0\rangle$ is the state with all spins $\boldsymbol{s}_{i}$ fully aligned along $-\boldsymbol{B}^{i}$ plus small corrections $\left(\boldsymbol{\lambda}^{i} \approx\right.$ $\left.\boldsymbol{B}^{i}+s J \cdot \boldsymbol{B}^{i} /|\boldsymbol{B}|^{i}\right)$. Up to first order in $\Delta_{ \pm} / \lambda$, Eqs. (10)-113) lead then to $Z^{i j} \approx V_{i j} \approx \frac{\Delta_{-}^{i j}}{\lambda_{i}+\lambda_{j}}$, entailing

$$
\left|0_{\mathrm{RPA}}\right\rangle \approx|0\rangle+\sum_{i<j} \frac{\Delta_{-}^{i j}}{\lambda_{i}+\lambda_{j}}\left|1_{i} 1_{j}\right\rangle,
$$

which, by Eq. (15), is just the first order expansion (in $\Delta_{-} / \Lambda$ ) of the exact ground state.

In the case of a symmetry-breaking mean field, the RPA spin state allows to implement the necessary rotations for symmetry restoration: The exact ground sate will actually be close to the superposition with the correct symmetry of the degenerate RPA ground states (rather than to a particular RPA state), as will be discussed in $\mathrm{Sec}$.IE in the context of parity-breaking. This restoration enlarges considerably the capabilities of the RPA.

\section{Bosonic evaluation of subsystem entropy and negativity}

The direct evaluation of many-body correlations and entanglement measures from the RPA spin state (14) is in general difficult. However, the values of these quantities in the associated bosonic vacuum (13), which will be close to those obtained from (14), can be straightforwardly evaluated using the general gaussian state formalism [25, 26]. The reduced density matrix of any subsystem is just a gaussian state, i.e., a canonical thermal state of an effective quadratic bosonic Hamiltonian, since Wick's theorem holds for the evaluation of the mean value of any observable, and in particular those of the subsystem. We may then evaluate its entropy and other invariants through standard expressions for independent boson systems.

Let us formalize the previous scheme. We will use a generalized contraction matrix formalism, equivalent to that based on covariance matrices [25, 26], which is more natural for the present RPA formulation. In the new vacuum $\left|0_{b}^{\prime}\right\rangle,\left\langle b_{\alpha}^{\prime \dagger} b_{\alpha^{\prime}}^{\prime}\right\rangle_{0^{\prime}}=\left\langle b_{\alpha}^{\prime} b_{\alpha^{\prime}}^{\prime}\right\rangle_{0^{\prime}}=0$, implying

$$
\begin{aligned}
F_{i j} & \equiv\left\langle b_{j}^{\dagger} b_{i}\right\rangle_{0^{\prime}}=\left(V V^{\dagger}\right)_{i j}, \\
G_{i j} & \equiv\left\langle b_{j} b_{i}\right\rangle_{0^{\prime}}=\left(V U^{\mathrm{tr}}\right)_{i j} .
\end{aligned}
$$

Eqs. (5) - (17) determine the basic RPA spin averages and correlations, i.e., $\left\langle s_{i \mu}\right\rangle_{0^{\prime}}=\delta_{\mu z}\left(F_{i i}-s_{i}\right)$ and, for $i \neq j$,

$$
\left\langle s_{i+} s_{j-}\right\rangle_{0^{\prime}}=2 \sqrt{s_{i} s_{j}} F_{j i},\left\langle s_{i-} s_{j-}\right\rangle_{0^{\prime}}=2 \sqrt{s_{i} s_{j}} G_{j i},
$$

with $\left\langle s_{i \pm} s_{j z}\right\rangle_{0^{\prime}}=0$, which coincide exactly with the averages derived from (14) up to second order in $V$, i.e., first order in the average occupation $V V^{\dagger}$ (normally very small outside critical regions). Through the use of Wick's theorem, we also obtain $\left\langle s_{i z} s_{j z}\right\rangle_{0^{\prime}}=\left\langle s_{i z}\right\rangle_{0^{\prime}}\left\langle s_{j z}\right\rangle_{0^{\prime}}+$ $\left|F_{i j}\right|^{2}+\left|G_{i j}\right|^{2}$ for $i \neq j$.

We may now define the generalized contraction matrix

$$
\mathcal{D} \equiv\left\langle\mathcal{Z} \mathcal{Z}^{\dagger}\right\rangle_{0^{\prime}}-\mathcal{M}=\left(\begin{array}{cc}
F & G \\
\bar{G} & I+\bar{F}
\end{array}\right),
$$

which exhibits the correct transformation rule under Bogoliubov transformations: If $\mathcal{Z}=\mathcal{W} \mathcal{Z}^{\prime}$, then

$$
\mathcal{D}=\mathcal{W} \mathcal{D}^{\prime} \mathcal{W}^{\dagger}
$$

with $\mathcal{D}^{\prime}=\left\langle\mathcal{Z}^{\prime} \mathcal{Z}^{\prime \dagger}\right\rangle_{0^{\prime}}-\mathcal{M}$. Eq. (17) can in fact be written in the form (201) if $\mathcal{W}$ is the diagonalizing Bogoliubov matrix (11) and $\mathcal{D}^{\prime}$ the vacuum density $\left(F^{\prime}=G^{\prime}=0\right)$. We may then also obtain $\mathcal{W}$ and $\mathcal{D}^{\prime}$ through the symplectic diagonalization of $\mathcal{D}$, i.e., through the diagonalization of

$$
\mathcal{D} \mathcal{M}=\left(\begin{array}{cc}
F & -G \\
\bar{G} & -I-\bar{F}
\end{array}\right)
$$

such that $\mathcal{W}^{-1} \mathcal{D} \mathcal{M} \mathcal{W}=\mathcal{D}^{\prime} \mathcal{M}$, with $\mathcal{D}^{\prime}$ diagonal.

Let us consider now a subsystem $A$ of $m<n$ sites. It will be characterized by a truncated contraction matrix

$$
\mathcal{D}_{A}=\left\langle\mathcal{Z}_{A} \mathcal{Z}_{A}^{\dagger}\right\rangle_{0_{b}^{\prime}}-\mathcal{M}_{A}=\left(\begin{array}{cc}
F_{A} & G_{A} \\
\bar{G}_{A} & I+\bar{F}_{A}
\end{array}\right)
$$

where $\mathcal{Z}_{A}$ contains just the bosons of sites in $A$. A symplectic diagonalization of $\mathcal{D}_{A}$ will lead to

$$
\mathcal{D}_{A}=\mathcal{W}_{A} \mathcal{D}^{\prime}{ }_{A} \mathcal{W}_{A}^{\dagger}, \quad \mathcal{D}_{A}^{\prime}=\left(\begin{array}{cc}
f_{A} & 0 \\
0 & I+f_{A}
\end{array}\right),
$$

where $f_{A}^{\alpha \alpha^{\prime}}=f_{A}^{\alpha} \delta^{\alpha \alpha^{\prime}}$ with $f_{A}^{\alpha}=\left\langle b^{\prime \dagger}{ }_{\alpha_{A}}^{\prime} b_{\alpha_{A}}^{\prime}\right\rangle_{0^{\prime}} \geq$ $0\left(\mathcal{D}_{A} \mathcal{M}_{A}\right.$ has eigenvalues $f_{A}^{\alpha}$ and $\left.-1-f_{A}^{\alpha}\right)$ and $\mathcal{W}_{A} \mathcal{M}_{A} \mathcal{W}_{A}^{\dagger}=\mathcal{M}_{A}$, with $\mathcal{Z}_{A}=\mathcal{W}_{A} \mathcal{Z}_{A}^{\prime}$. The entanglement between $A$ and its complement $A$ is then given by the associated bosonic entropy,

$$
\begin{aligned}
S\left(\rho_{A}^{b}\right) & =-\operatorname{Tr} \rho_{A}^{b} \log _{2} \rho_{A}^{b} \\
& =-\sum_{\alpha} f_{A}^{\alpha} \log _{2} f_{A}^{\alpha}-\left(1+f_{A}^{\alpha}\right) \log _{2}\left(1+f_{A}^{\alpha}\right)
\end{aligned}
$$


Here $\rho_{A}^{b} \equiv \operatorname{Tr}_{\bar{A}}\left|0_{b}^{\prime}\right\rangle\left\langle 0_{b}^{\prime}\right|$ is the bosonic reduced density of subsystem $A$, which can be explicitly written as

$$
\rho_{A}^{b}=C \exp \left[-\frac{1}{2} \mathcal{Z}_{A}^{\dagger} \mathcal{H}_{A} \mathcal{Z}_{A}\right]=C \exp \left[-\sum_{\alpha} \omega_{A}^{\alpha}{b^{\prime}}_{\alpha_{A}}^{\dagger} b_{\alpha}^{\prime}\right.
$$

where $C=\prod_{\alpha}\left(1+f_{\alpha}^{A}\right)$ and $\mathcal{H}_{A}, \mathcal{D}_{A}$ are related by

$$
\mathcal{D}_{A} \mathcal{M}_{A}=\left[\exp \left(\mathcal{M}_{A} \mathcal{H}_{A}\right)-I\right]^{-1} \text {. }
$$

Here $\mathcal{H}_{A}$ represents an effective "Hamiltonian" matrix for subsystem $A$ with symplectic eigenvalues $\omega_{A}^{\alpha}$ such that $f_{A}^{\alpha}=\left(e^{\omega_{A}^{\alpha}}-1\right)^{-1}$ (and hence $\left.-1-f_{A}^{\alpha}=\left(e^{-\omega_{A}^{\alpha}}-1\right)^{-1}\right)$. Eq. (26) leads then to the contraction matrix (22), and hence to the same expectation values as the full vacuum $\left|0_{b}^{\prime}\right\rangle\left\langle 0_{b}^{\prime}\right|$ for any operator of subsystem $A$.

Eq. (25) provides a tractable RPA estimation of the entanglement entropy of any subsystem. It is shown in the Appendix $\mathrm{A}$ that a direct spin evaluation of the subsystem entropy based on the RPA state (14) coincides with (25) up to second order in $V$.

On the other hand, the internal entanglement of subsystem $A$ with respect to a partition $(B, C)$ of $A$ (where the complement $\bar{A}$ plays the role of an environment) can be measured through the corresponding negativity [13], defined as minus the sum of the negative eigenvalues of the partial transpose $\rho_{A}^{t_{C}}$ of $\rho_{A}$ :

$$
N_{B C}=\frac{1}{2}\left(\operatorname{Tr}\left|\rho_{A}^{t_{C}}\right|-1\right) .
$$

Expectation values with respect to $\left(\rho_{A}^{b}\right)^{t_{C}}$ of an observable $O_{A}^{b}$ correspond to those of the partial transpose $\left(O_{A}^{b}\right)^{t_{C}}$ with respect to $\rho_{A}^{b}$. This implies the replacements $F_{i j} \leftrightarrow G_{i j}, F_{j^{\prime} j} \leftrightarrow F_{j j^{\prime}}, G_{j^{\prime} j} \leftrightarrow \bar{G}_{j^{\prime} j}$, in the contraction matrix for $j, j^{\prime} \in C, i \in B$, leading to a matrix $\tilde{\mathcal{D}}_{A}$ with symplectic eigenvalues $\tilde{f}_{A}^{\alpha}$. The latter can now be negative. We may then still write $\left(\rho_{A}^{b}\right)^{t_{C}}$ as in Eq. (26) in terms of an effective matrix $\tilde{\mathcal{H}}_{A}$ with symplectic eigenvalues $\tilde{\omega}_{A}^{\alpha}$ such that $\tilde{f}_{A}^{\alpha}=\left(e^{\tilde{\omega}_{A}^{\alpha}}-1\right)^{-1}$.

Since the trace remains unchanged $\left(\operatorname{Tr}\left(\rho_{A}^{b}\right)^{t_{C}}=1\right)$, $\left|e^{-\tilde{\omega}_{A}^{\alpha}}\right|<1$, implying $\tilde{f}_{A}^{\alpha}>-1 / 2$. A negative $\tilde{f}_{A}^{\alpha}>-1 / 2$ corresponds to $e^{-\tilde{\omega}_{A}^{\alpha}}<0$ and hence to a non-positive $\left(\rho_{A}^{b}\right)^{t_{C}}$, indicating an entangled $\rho_{A}^{b}$ with respect to this bipartition. We then obtain, noting that $\left(1+e^{-\tilde{\omega}_{A}^{\alpha}}\right)^{-1}=$ $\left(1+\tilde{f}_{A}^{\alpha}\right) /\left(1+2 \tilde{f}_{A}^{\alpha}\right)$, the final result [13, 25, 26]

$$
\operatorname{Tr}\left|\left(\rho_{A}^{b}\right)^{t_{c}}\right|=\prod_{\tilde{f}_{A}^{\alpha}<0} \frac{1}{1+2 \tilde{f}_{A}^{\alpha}},
$$

which allows the evaluation of the negativity (28). Negativities obtained from the spin density matrices coincide with this result up to first order in $V$ (Appendix A).

In the case of a global bipartition $(A, \bar{A}), N_{A \bar{A}}$ becomes a function of the reduced density $\rho_{A}$, namely [17]

$$
\left.N_{A \bar{A}}=\frac{1}{2}(\operatorname{Tr}|| 0\rangle\left\langle\left. 0\right|^{\mathrm{t}_{\bar{A}}}\right|-1\right)=\frac{1}{2}\left[\left(\operatorname{Tr} \sqrt{\rho_{A}}\right)^{2}-1\right] .
$$

In a boson system, this implies that $N_{A \bar{A}}$, a limit case of Eqs. (28)-(29), can be also expressed just in terms of the symplectic eigenvalues $f_{A}^{\alpha}$ of the contraction matrix $\mathcal{D}_{A}$ :

$$
N_{A \bar{A}}=\frac{1}{2}\left[\prod_{\alpha}\left(\sqrt{f_{A}^{\alpha}}+\sqrt{1+f_{A}^{\alpha}}\right)^{2}-1\right] .
$$

\section{Translationally invariant systems}

The only quantities required in the bosonic RPA scheme are, therefore, the basic contractions (17). Their evaluation becomes remarkably simple in translationally invariant systems, either in one or $d$ dimensions, i.e., systems with a common spin $s_{i}=s$ in a uniform field $\boldsymbol{B}^{i}=\boldsymbol{B}$ with couplings dependent just on separation:

$$
J^{i \mu j \nu}=J^{\mu \nu}(i-j)
$$

where $J^{\mu \nu}(l)=J^{\nu \mu}(-l)$, and $J^{\mu \nu}(-l)=J^{\mu \nu}(n-l)$ in a finite cyclic chain or system (in $d$ dimensions, $i, j, l, n$ stand for $d$-dimensional vectors). We will also assume a uniform mean field $\boldsymbol{\lambda}^{i}=\boldsymbol{\lambda}$, which should then satisfy

$$
\lambda^{\mu}=B^{\mu}-\sum_{\nu} J_{0}^{\mu \nu}\left\langle s_{\nu}\right\rangle_{0}, \quad J_{0}^{\mu \nu} \equiv \sum_{l} J^{\mu \nu}(l),
$$

with $\langle s\rangle_{0}=-s \boldsymbol{\lambda} / \lambda$ (Eq. (4)). The uniform mean field is thus determined just by the total strengths $J_{0}^{\mu \nu}$.

Choosing again the $z$ axis in the direction of $\boldsymbol{\lambda}$, such that $\left\langle s_{i \mu}\right\rangle=-s \delta_{\mu z}$ and $B^{\mu}+s J_{0}^{\mu z}=\lambda \delta^{\mu z}$, with $\lambda>0$, the bosonized Hamiltonian will have the form (6) with couplings $\Delta_{ \pm}^{i j}=\Delta_{ \pm}(i-j)$. By means of a discrete Fourier transform of the boson operators, we can rewrite it as

$$
\begin{aligned}
H_{b} & =\langle H\rangle_{0}+\sum_{k}\left(\lambda-\Delta_{+}^{k}\right) b_{k}^{\dagger} b_{k}-\frac{1}{2}\left(\Delta_{-}^{k} b_{k}^{\dagger} b_{-k}^{\dagger}+h .\right. \\
\Delta_{ \pm}^{k} & =\sum_{l=0}^{n-1} e^{i 2 \pi k l / n} \Delta_{ \pm}(l)
\end{aligned}
$$

where $k=0, \ldots, n-1$ and $b_{k}=\frac{1}{\sqrt{n}} \sum_{j=1}^{n} e^{i 2 \pi k j / n} b_{j}$ are boson operators in momentum space, with $b_{-k}=b_{n-k}$. Diagonalization of (34) is straightforward and leads to

$$
H^{b}=\langle H\rangle_{0}+\sum_{k} \omega^{k} b^{\prime \dagger} b_{k}^{\prime}+\frac{1}{2}\left(\omega^{k}-\lambda+\Delta_{+}^{k}\right),
$$

where $\omega^{k}=\tilde{\omega}^{k}-\frac{1}{2}\left(\Delta_{+}^{k}-\Delta_{+}^{-k}\right),{b^{\prime}}_{k}^{\dagger}=u_{k} b_{k}^{\dagger}+\bar{v}_{k} b_{-k}$ and

$$
\begin{aligned}
& \tilde{\omega}^{k}=\sqrt{\left(\lambda-\tilde{\Delta}_{+}^{k}\right)^{2}-\left|\Delta_{-}^{k}\right|^{2}}, \\
& u_{k}=\sqrt{\frac{\lambda-\tilde{\Delta}_{+}^{k}+\tilde{\omega}_{k}}{2 \tilde{\omega}^{k}}}, v_{k}=\frac{\Delta_{-}^{k}}{\left|\Delta_{-}^{k}\right|} \sqrt{\frac{\lambda-\tilde{\Delta}_{+}^{k}-\tilde{\omega}_{k}}{2 \tilde{\omega}^{k}}}(38)
\end{aligned}
$$

with $\tilde{\Delta}_{+}^{k}=\frac{1}{2}\left(\Delta_{+}^{k}+\Delta_{+}^{-k}\right), u_{k}^{2}-\left|v_{k}\right|^{2}=1$, and $u_{k}=u_{-k}$, $v_{k}=v_{-k}$. All $\omega^{k}$ should be real and positive for a stable mean field, implying the stability conditions

$$
0 \leq\left|\Delta_{-}^{k}\right|<\lambda-\Delta_{+}^{k}, \quad k=0, \ldots, n-1 .
$$


We can now obtain the basic contractions explicitly,

$$
\left\langle b_{k}^{\dagger} b_{k^{\prime}}\right\rangle_{0^{\prime}}=\delta_{k k^{\prime}}\left|v_{k}^{2}\right|,\left\langle b_{k} b_{-k^{\prime}}\right\rangle_{0^{\prime}}=\delta_{k k^{\prime}} u_{k} v_{k}=\frac{\Delta_{-}^{k}}{2 \tilde{\omega}^{k}}
$$

which lead finally to (Eq. (17))

$$
\begin{gathered}
F_{i j}=F(i-j)=\frac{1}{n} \sum_{k} e^{-i 2 \pi k(i-j) / n}\left|v_{k}^{2}\right|, \\
G_{i j}=G(i-j)=\frac{1}{n} \sum_{k} e^{-i 2 \pi k(i-j) / n} u_{k} v_{k} .
\end{gathered}
$$

For strong fields $|B|$ such that $\lambda \gg\left|\Delta_{ \pm}\right|, u_{k} v_{k} \approx \frac{1}{2} \Delta_{-}^{k} / \lambda$ and $\left|v_{k}^{2}\right| \approx \frac{1}{4}\left|\Delta_{-}^{k}\right|^{2} / \lambda^{2}$. The RPA vacuum (13) becomes

$$
\left|0_{b}^{\prime}\right\rangle=C_{b} \exp \left[\frac{1}{2} \sum_{i, j} Z(i-j) b_{i}^{\dagger} b_{j}^{\dagger}\right]\left|0_{b}\right\rangle
$$

where $C_{b}=\prod_{k} u_{k}^{-1 / 2}$ and $Z(l)=\frac{1}{n} \sum_{k} e^{-i 2 \pi l k / n} \frac{v_{k}}{u_{k}}$.

Thus, these systems allow an analytic evaluation of the contractions (17). Both the mean field equations (33) and the RPA Hamiltonian (34) become independent of the common spin $s$ after a rescaling $J^{\mu \nu}(l) \rightarrow J^{\mu \nu}(l) / s$, which we will adopt in what follows and which indicates that RPA is describing the large spin limit of the system, as is apparent from Eq. (5).

\section{E. XYZ systems}

Let us now examine in more detail the previous formalism in a translationally invariant spin $s$ array with $X Y Z$ couplings of arbitrary range in a uniform transverse field:

$$
H=B \sum_{i} s_{i z}-\frac{1}{2 s} \sum_{i \neq j} \sum_{\mu=x, y, z} J_{\mu}(i-j) s_{i \mu} s_{j \mu} .
$$

Eq. (43) commutes with the $S_{z}$ spin parity,

$$
\left[H, P_{z}\right]=0, \quad P_{z}=\exp \left[i \pi \sum_{i}\left(s_{i z}-s\right)\right],
$$

for any value of its parameters, such that the exact ground state in a finite array will always have a definite parity outside degeneracy points. We will focus here on the ferromagnetic type case where $J_{x}(l) \geq 0 \forall l$ with

$$
\left|J_{y}(l)\right| \leq J_{x}(l)
$$

which exhibits a normal and parity breaking phase at the mean field level.

\section{RPA around the normal state}

For the Hamiltonian (43), the state $|0\rangle$ with all spins fully aligned along the $-z$ axis is always a solution of the mean field equation (33), being the lowest solution for a sufficiently strong field $B$. It leads to $\lambda^{i}=\lambda \delta^{\mu z}$, with

$$
\lambda=|B|+J_{z}^{0}>0, \quad J_{z}^{0} \equiv \sum_{l} J_{z}(l)
$$

All previous equations can then be directly applied. Now $\Delta_{ \pm}(l)=\frac{J_{x}(l) \pm J_{y}(l)}{2}=\Delta_{ \pm}(-l)$, implying $\Delta_{ \pm}^{k}=\Delta_{ \pm}^{-k}$ and

$$
\omega^{k}=\sqrt{\left(\lambda-J_{x}^{k}\right)\left(\lambda-J_{y}^{k}\right)}
$$

where $J_{\mu}^{k}=\sum_{l} e^{i 2 \pi k l / n} J_{\mu}(l)\left(\Delta_{ \pm}^{k}=\frac{J_{x}^{k} \pm J_{y}^{k}}{2}\right)$. This solution is therefore stable provided $J_{\mu}^{k} \leq \lambda \forall k$ and $\mu=x, y$, i.e. for $|B|$ above a certain critical field $B_{c}$. In the case (44), the strongest condition is obtained for $k=0$, i.e.,

$$
|B|>B_{c} \equiv J_{x}^{0}-J_{z}^{0}
$$

\section{RPA around the parity breaking state}

For $|B| \leq B_{c}$, the normal state becomes unstable: the lowest normal RPA frequency $\omega^{0}$ vanishes for $|B| \rightarrow B_{c}$ and becomes imaginary for $|B|<B_{c}$. The lowest mean field for $|B|<B_{c}$ corresponds instead to a paritybreaking state with all spins aligned along an axis in the $x z$ plane forming an angle $\theta$ with the $z$ axis:

$$
|0\rangle \rightarrow|\Theta\rangle \equiv\left|\theta_{1} \ldots \theta_{n}\right\rangle, \quad\left|\theta_{j}\right\rangle=\exp \left[-i \theta s_{j y}\right]\left|0_{j}\right\rangle .
$$

This leads to $\left\langle\boldsymbol{s}_{j}\right\rangle_{0}=-s(\sin \theta, 0, \cos \theta)=-s \boldsymbol{\lambda} / \lambda$, with

$$
\lambda=J_{x}^{0}, \quad \cos \theta=B / B_{c},
$$

as determined by (33). We should now express the original spin operators in terms of the rotated operators, i.e.,

$$
s_{i x}=s_{i x^{\prime}} \cos \theta+s_{i z^{\prime}} \sin \theta, \quad s_{i z}=s_{i z^{\prime}} \cos \theta-s_{i x^{\prime}} \sin \theta
$$

with $s_{i y}=s_{i y^{\prime}}$. The RPA around this state amounts therefore to the replacements

$$
\lambda \rightarrow J_{x}^{0}, \quad J_{x}^{k} \rightarrow J_{x}^{\prime k}=J_{x}^{k} \cos ^{2} \theta+J_{z}^{k} \sin ^{2} \theta
$$

in Eq. (46), with $J_{y}^{k}$ unchanged and $\Delta_{ \pm}^{k}=\frac{1}{2}\left(J_{x}^{\prime k} \pm J_{y}^{k}\right)$.

Correlations $\left\langle s_{i \mu^{\prime}} s_{j \mu^{\prime}}\right\rangle_{\mathrm{RPA}}$ of rotated spin operators have the same previous expressions (17), whereas those of the original operators must be obtained using Eqs. (50). It should be remarked, however, that in a finite system, the associated RPA spin state will no longer be a good approximation to the actual ground state due to parity breaking. Parity restoration, at least approximately, must be implemented before obtaining final results. We will not discuss here the case of a continuous broken symmetry (arising for instance in the $X X Z$ case), which can be treated through the RPA formalism of ref. [9]. 


\section{Definite Parity RPA ground states}

Since $\left[H, P_{z}\right]=0$, the parity breaking mean field state $|\Theta\rangle$ is degenerate: Both $|\Theta\rangle$ and $|-\Theta\rangle=P_{z}|\Theta\rangle$ are mean field ground states. In order to describe the definite parity ground states, the correct RPA ground state should be taken as the definite parity combinations

$$
\left|\Theta_{\mathrm{RPA}}^{ \pm}\right\rangle=\frac{\left|\Theta_{\mathrm{RPA}}\right\rangle \pm\left|-\Theta_{\mathrm{RPA}}\right\rangle}{\sqrt{2\left(1 \pm\left\langle-\Theta_{\mathrm{RPA}} \mid \Theta_{\mathrm{RPA}}\right\rangle\right)}},
$$

where $\left| \pm \Theta_{\mathrm{RPA}}\right\rangle$ are the RPA states around each mean field. The overlap $\left\langle-\Theta_{\mathrm{RPA}} \mid \Theta_{\mathrm{RPA}}\right\rangle=\left\langle\Theta_{\mathrm{RPA}}\left|P_{z}\right| \Theta_{\mathrm{RPA}}\right\rangle$ is proportional to the overlap between the two mean fields,

$$
\langle-\Theta \mid \Theta\rangle=\cos ^{2 n s} \theta=\left(B / B_{c}\right)^{2 n s},
$$

which is small except for $B \rightarrow B_{c}$ or small $n s$.

Neglecting the previous overlap, Eq. (52) will lead to reduced densities

$$
\rho_{A}^{ \pm} \approx \frac{1}{2}\left[\rho_{A}(\theta)+\rho_{A}(-\theta)\right]
$$

provided the complementary overlap $\left\langle-\Theta_{\mathrm{RPA}}^{\bar{A}} \mid \Theta_{\mathrm{RPA}}^{\bar{A}}\right\rangle \propto$ $\left(\frac{B}{B_{c}}\right)^{2\left(n-n_{A}\right) s}$ can also be neglected. Here $\rho_{A}( \pm \Theta)$ are the reduced spin densities determined by each RPA state, given up to $O\left(V^{2}\right)$ by the expressions of Appendix A.

The restoration (54) is essential to achieve a good description of the actual subsystem entropy, although its main effect for a not too small subsystem $A$ is actually quite simple: If the product $\rho_{A}(\Theta) \rho_{A}(-\Theta) \propto$ $\left(B / B_{c}\right)^{2 n_{A} s}$ can be neglected, Eq. (54) can be considered as the sum of two densities with orthogonal support and identical distributions, leading to

$$
S\left(\rho_{A}^{ \pm}\right) \approx S\left(\rho_{A}(\theta)\right)+1,
$$

where $S\left(\rho_{A}(\Theta)\right)$ can be evaluated through the boson approximation (25). Under the same assumptions, the effect on the global negativity (30) is just

$$
N_{A \bar{A}}\left(\rho_{A}^{ \pm}\right) \approx 2 N_{A \bar{A}}\left(\rho_{A}(\theta)\right)+\frac{1}{2},
$$

as $\operatorname{Tr} \sqrt{\rho_{A}^{ \pm}} \approx \sqrt{2} \operatorname{Tr} \sqrt{\rho_{A}(\theta)}$, while the subsystem negativity $N_{B C}$ of a bipartition $(B, C)$ of $A$ remains approximately unchanged: $N_{B C}\left(\rho_{A}^{ \pm}\right) \approx N_{B C}\left(\rho_{A}(\theta)\right)$.

When the product $\rho_{A}(\Theta) \rho_{A}(-\Theta)$ cannot be neglected (as in a subsystem of two spins), we should in principle construct the spin density (54). This can be done by rotating $\rho_{A}(\theta)$ (Eq. (A2) in the mean field frame) to the original $z$ axis and removing all parity breaking elements (which is the final effect of Eq. (54)). For instance, the reduced two-spin density for $s=1 / 2$ has the blocked form (A2) in the standard basis of $s_{i z} s_{j z}$ eigenstates in the normal phase as well as in the parity breaking phase after parity restoration [12]. The final effect on $S\left(\rho_{A}\right)$ is the replacement of the term +1 in (55) by the entropy of the reduced mean field mixture $-\sum_{\nu= \pm} q_{\nu} \log _{2} q_{\nu}$, with $q_{ \pm}=\frac{1}{2}\left(1 \pm\left(B / B_{C}\right)^{2 s_{A}}\right)$, plus small RPA corrections.
While $\rho_{A}^{ \pm}$are both identical in the approximation (54), the actual $\rho_{A}^{ \pm}$in a small system will depend on parity. The correct parity in such a case should be chosen as that leading to the lowest energy $E_{\mathrm{RPA}}^{ \pm}=\left\langle\Theta_{\mathrm{RPA}}^{ \pm}|H| \Theta_{\mathrm{RPA}}^{ \pm}\right\rangle$.

\section{Factorizing Field}

The explicit value of the basic RPA couplings $\Delta_{ \pm}^{k}$ in the parity breaking phase are, using Eqs. (51)-(49),

$$
\left.\Delta_{ \pm}^{k}=\frac{1}{2}\left[\left(J_{x}^{k}-J_{z}^{k}\right)\left(B / B_{c}\right)^{2}+J_{z}^{k} \pm J_{y}^{k}\right)\right]
$$

In the case of a common anisotropy, such that the ratio

$$
\chi=\frac{J_{y}(l)-J_{z}(l)}{J_{x}(l)-J_{z}(l)}
$$

is independent of the separation $l$, we have $J_{y}^{k}-J_{z}^{k}=$ $\chi\left(J_{x}^{k}-J_{z}^{k}\right)$ and hence $\Delta_{-}^{k}=\frac{1}{2}\left(J_{x}^{k}-J_{z}^{k}\right)\left[\left(B / B_{c}\right)^{2}-\chi\right]$. It is then seen that if $\chi \in[0,1], \Delta_{-}^{k}=0 \forall k$ when

$$
|B|=B_{s} \equiv B_{c} \sqrt{\chi}
$$

with all $\Delta_{-}^{k}$ changing sign at $|B|=B_{s}$. Here $B_{s}$ is the factorizing field [2, 12, 22 24]: At $B=B_{s}$ the parity breaking mean field state becomes an exact ground state, since the RPA corrections vanish (sec. IIB). This effect is independent of the number of spins $n$ (as long as $\chi$ is constant) and spin $s$ (with the present scaling). Nonetheless, the actual side limits at $B=B_{s}$ will be given by the definite parity states (52), which are still entangled. As a consequence, the subsystem entropy $S\left(\rho_{A}\right)$ and the negativity $N_{A \bar{A}}$ will actually approach a finite value for $B \rightarrow B_{s}$ (1 and $1 / 2$ respectively in the approximation (55) - (56) ), while the entanglement between two spins will reach there infinite range [10 12]. Note finally that at $B=B_{s}, \Delta_{+}^{k}=J_{y}^{k}$ and hence,

$$
\omega^{k}=J_{x}^{0}-J_{y}^{k} .
$$

\section{APPLICATION}

\section{A. Spin $s$ pair}

As a first example, let us consider a system of two spins $s$ coupled through the Hamiltonian (43). We can obviously always set here $J_{x} \geq\left|J_{y}\right|$ (Eq. (44)), since the sign of $J_{x}$ can be changed by a $\pi$-rotation around the $z$ axis of one of the spins (and we can always set $\left|J_{x}\right| \geq\left|J_{y}\right|$ by a proper choice of axes). The Fourier transform of $J_{\mu}(l)=\delta_{l 1} J_{\mu}$ reduces here to $J_{\mu}^{k}=(-1)^{k} J_{\mu}, k=0,1$, leading to an attractive and a repulsive normal mode:

$$
\omega_{0}=\sqrt{\left(\lambda-J_{x}\right)\left(\lambda-J_{y}\right)}, \omega_{1}=\sqrt{\left(\lambda+J_{x}\right)\left(\lambda+J_{y}\right)} .
$$

The contractions (41) become $F_{i j}=\frac{\lambda-\Delta_{+}}{4 \omega_{0}}-\frac{\lambda+\Delta_{+}}{4 \omega_{1}}(1-$ $\left.2 \delta_{i j}\right)-\frac{1}{2} \delta_{i j}, G_{i j}=\frac{\Delta_{-}}{4 \omega_{0}}+\frac{\Delta_{-}}{4 \omega_{1}}\left(1-2 \delta_{i j}\right)$, where $\Delta_{ \pm}=$ 


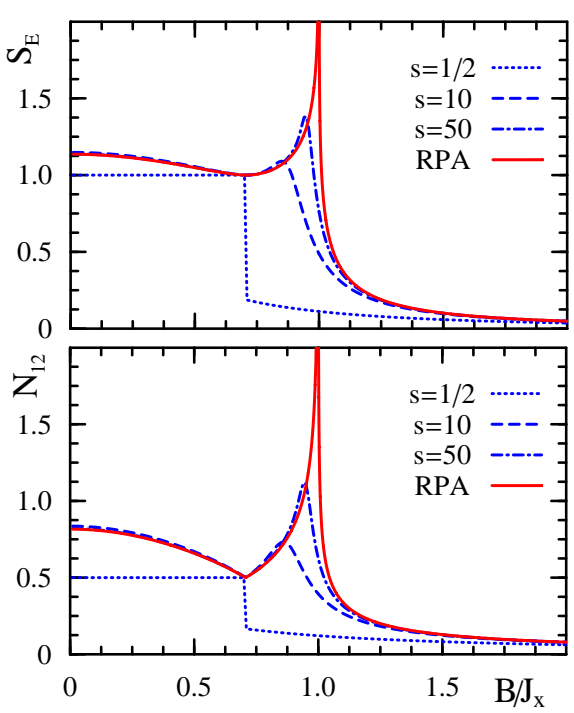

FIG. 1. Entanglement between two spins $s$ as a function of the transverse field $B$ for an $X Y$ coupling with $J_{y} / J_{x}=0.5$. The exact entanglement entropy $S_{E}=S\left(\rho_{1}\right)$ (top) and negativity (bottom) for different values of the spin $s$, and the bosonic RPA results, Eqs. (61), (63) are depicted. The exact results approach those of RPA as $s$ increases, differences for not too small $s$ arising just for $B$ close to $B_{c}=J_{x}$. At the factorizing field $B_{s} \approx B_{c} / \sqrt{2}, S_{E}=1$ while $N_{12}=1 / 2$.

$\frac{1}{2}\left(J_{x} \pm J_{y}\right)$ and replacements (51) are to be applied for $|B|<B_{c}$. The ensuing entanglement entropy of the pair in the bosonic approximation (25) is just

$$
\begin{aligned}
S\left(\rho_{1}\right) & =-f \log _{2} f+(1+f) \log _{2}(1+f)+\delta, \\
f & =\frac{1}{2}\left(\sqrt{1+\frac{\lambda^{2}-\bar{\omega}^{2}}{\omega_{0} \omega_{1}}}-1\right), \quad \bar{\omega}=\frac{\omega_{0}+\omega_{1}}{2}
\end{aligned}
$$

where $f=\sqrt{\left(F_{11}+\frac{1}{2}\right)^{2}-\left(G_{11}\right)^{2}}-\frac{1}{2}$ is the positive symplectic eigenvalue of the $2 \times 2$ contraction matrix for one spin and $\delta=0(1)$ for $|B|>B_{c}\left(<B_{c}\right)$ in the approximation (55), valid for $\left.\left(B / B_{c}\right)^{2 s} \ll 1\right)$. For small $f$, we may just use $S\left(\rho_{1}\right) \approx f\left(\log _{2} e-\log _{2} f\right)$, with $f \approx F_{11}$, in agreement with the results of Appendix A.

Thus, at the RPA level entanglement is determined by the average local occupation $f$ and driven by the ratio $\frac{\lambda^{2}-\bar{\omega}^{2}}{\omega_{0} \omega_{1}}$, which is small away from $B_{c}$ and vanishes at $B=$ $B_{s}$ (where $\bar{\omega}=\lambda=J_{x}^{0}$ by Eq. (60), and hence $f=0$ ). For $|B| \gg B_{c}, f \approx\left(\frac{J_{x}-J_{y}}{4 B}\right)^{2}$, while in the vicinity of $B_{s}, f \propto\left(B-B_{s}\right)^{2}$. For $B \rightarrow B_{c}, f \approx \frac{1}{2} \sqrt{\frac{\lambda^{2}-\bar{\omega}^{2}}{\omega_{0} \omega_{1}}} \propto$ $\left|B-B_{c}\right|^{-1 / 4}$, with $S\left(\rho_{1}\right) \approx \log _{2} f e$.

The bosonic RPA negativity (28)-(29) becomes

$$
N_{12}=\frac{-\tilde{f}}{1+2 \tilde{f}}=f+\sqrt{f(f+1)}
$$

where $\tilde{f}=f-\sqrt{f(f+1)}$ is the negative symplectic eigenvalue of the $4 \times 4$ contraction matrix. Correction
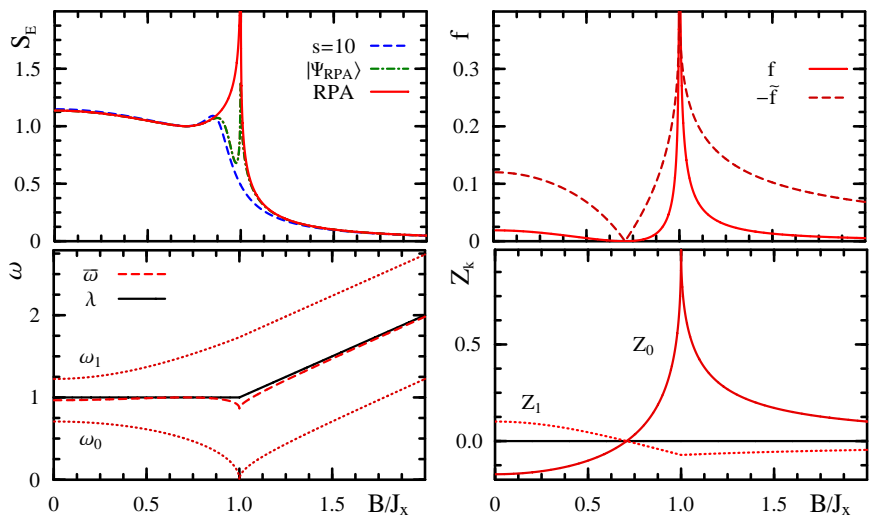

FIG. 2. Top: Left: The entanglement entropy obtained from the definite parity RPA spin state (52) (dashed-dotted line), compared with the bosonic RPA result (61) and the exact value, for $s=10$ at the same parameters of fig. 1 The result from the RPA spin state improves the bosonic RPA for $B$ just below $B_{c}$. Right: The average local boson occupation $f$, Eq. (62), which is small away from $B_{c}$, and the negative eigenvalue $\tilde{f}$ of the partial transpose of the contraction matrix $(\tilde{f} \approx \sqrt{f}$ for small $f$ ). Bottom: Left: RPA energies $\omega_{0}, \omega_{1}$, together with the mean field energy $\lambda$ and the mean RPA energy $\bar{\omega}$ appearing in (62). Right: The quantities $Z_{k}=v_{k} / u_{k}$ for $k=0,1$, which determine the RPA state (42) and vanish at the factorizing field $B_{s}$.

(56) $\left(N_{21} \rightarrow 2 N_{21}+\frac{1}{2}\right)$ should be applied for $|B|<B_{c}$. For small $f$, we have simply $N_{12} \approx-\tilde{f} \approx \sqrt{f}$. This will lead to a slope discontinuity of $N_{12}$ at the factorizing field $B_{s}$ (see Fig. 1), as $f$ vanishes there quadratically $\left(N_{12}-\frac{1}{2} \propto\left|B-B_{s}\right|\right.$ for $\left.B \approx B_{s}\right)$. On the other hand, for $f \rightarrow \infty\left(|B| \rightarrow B_{c}\right), \tilde{f} \rightarrow-\frac{1}{2}$, with $\tilde{f} \approx-\frac{1}{2}+\frac{1}{8 f}$ and $N_{12} \approx 2 f$. Both $S\left(\rho_{1}\right)$ and $N_{12}$ are concave increasing functions of $f$ and measure the entanglement of the pair.

Comparison with exact numerical results, obtained through the diagonalization of $H\left(\mathrm{a}(2 s+1)^{2} \times(2 s+1)^{2}\right.$ matrix), are shown in Fig. 1 for the $X Y$ case $\left(J_{z}=0\right)$ with anisotropy $\chi=J_{y} / J_{x}=0.5$. Exact results are seen to rapidly approach the RPA values (61) - (63) as the spin $s$ increases, the discrepancy for finite $s$ arising just in the vicinity of $B_{c}$ or for very small $s$, i.e., where tunneling effects arising from the non-zero overlap (53) between the degenerate parity breaking states become appreciable.

Nonetheless, this overlap can be taken into account using the full definite parity RPA spin state (52) with lowest energy, which for finite $s$ improves results for $B$ close to $B_{c}$ (but otherwise yields results almost coincident with those of the corrected bosonic RPA), as seen in fig. 2. Eq. (52) also yields the exact side limits at the factorizing field [12] for any $s$, although for $\chi=0.5$ these limits rapidly approach the high spin values $S\left(\rho_{1}\right)=1$ and $N_{12}=\frac{1}{2}$ predicted by the approximations (55)- (56) .

Fig. 2 also depicts the behavior of the average occupations $f$ and $\tilde{f}$. The former is seen to be quite small $(f \lesssim 0.05)$ except in the vicinity of $B_{c}$, implying that 
away from $B_{c}$, all bosonic RPA results can be reproduced by the spin densities of Appendix A, with $\tilde{f} \approx \sqrt{f}$. In the bottom panels we depict the RPA energies $\omega_{0}, \omega_{1}$ and the RPA state coefficients $Z_{k} \equiv v_{k} / u_{k}$ used in Eq. (42). Although $\omega_{0}$ vanishes at $B_{c}$, the difference $\lambda-\bar{\omega}$, responsible for entanglement, remains everywhere quite small. Both $Z_{k}$ vanish and change sign at the factorizing field $B_{s}$, indicating a qualitative change in the type of correlations at this point: Entanglement between two spins 1/2 is well known to change from antiparallel to parallel (in the original frame) at $B_{s}$ [12], an effect arising within the RPA from this sign change.

\section{B. Fully connected spin system}

Let us now consider a fully and uniformly connected $X Y Z$ array of $n$ spins, where

$$
J_{\mu}(l)=\left(1-\delta_{l 0}\right) J_{\mu} /(n-1),
$$

in (43). This scaling ensures a finite intensive energy $\langle H\rangle / n$ for large $n$ and finite $J_{\mu}$. Entanglement properties of this well-known model [18, 27] for $s=1 / 2$ in the large $n$ limit have been previously analyzed [28], including recently Holstein-Primakoff based bosonization [16, 21, 29, 30]. Direct application of the present RPA formalism will be here shown to yield full analytic expressions for any size $n$ and spin $s$. The present treatment does not exactly coincide with that of refs. [16, 21], since the absence of self-interacting terms $\propto s_{i \mu} s_{i \nu}$ (nontrivial for $s>1 / 2$ ) is here exactly taken into account and leads to repulsive RPA corrections $\left(\omega_{1}\right)$, non-zero for finite $n$. The Fourier transform of (64) is $J_{\mu}^{0}=J_{\mu}$ and $J_{\mu}^{k}=-J_{\mu} /(n-1)$ for $k=1, \ldots, n-1$, leading again to two distinct RPA energies: One associated with a fundamental attractive mode $\left(\omega_{0}\right)$ and $n-1$ degenerate weak repulsive modes $\omega_{k}=\omega_{1}, k \neq 0$, which just add a small repulsive correction accounting for the absence of selfenergy terms:

$$
\omega_{0}=\sqrt{\left(\lambda-J_{x}\right)\left(\lambda-J_{y}\right)}, \omega_{1}=\sqrt{\left(\lambda+\frac{J_{x}}{n-1}\right)\left(\lambda+\frac{J_{y}}{n-1}\right)}
$$

where the replacements (51) are to be used for $B<B_{c}$. The ensuing contractions (41) become here obviously independent of separation for $i \neq j$ :

$$
\begin{aligned}
F_{i j} & =\frac{1}{2 n}\left[\frac{\lambda-\Delta_{+}^{0}}{\omega_{0}}-\frac{\lambda-\Delta_{+}^{1}}{\omega_{1}}\left(1-n \delta_{i j}\right)\right]-\frac{1}{2} \delta_{i j}, \\
G_{i j} & =\frac{1}{2 n}\left[\frac{\Delta_{-}^{0}}{\omega_{0}}-\frac{\Delta_{-}^{1}}{\omega_{1}}\left(1-n \delta_{i j}\right)\right],
\end{aligned}
$$

and imply that for any bipartition $(L, n-L)$, the entanglement entropy $S\left(\rho_{L}\right)$ will depend just on $L$. Moreover, there is again a single non-zero eigenvalue $f_{L}$ of the reduced matrix $\mathcal{D}_{L}$ of $L$ spins for any $L$ (see Appendix B), such that in the bosonic approximation (25) (55),

$$
\begin{aligned}
S\left(\rho_{L}\right) & =-f_{L} \log _{2} f_{L}+\left(1+f_{L}\right) \log _{2}\left(1+f_{L}\right)+\delta,(66) \\
f_{L} & =\frac{1}{2}\left[\sqrt{1+2 \alpha_{L} \Delta}-1\right], \quad \alpha_{L}=L(n-L) / n^{2}(, 67)
\end{aligned}
$$
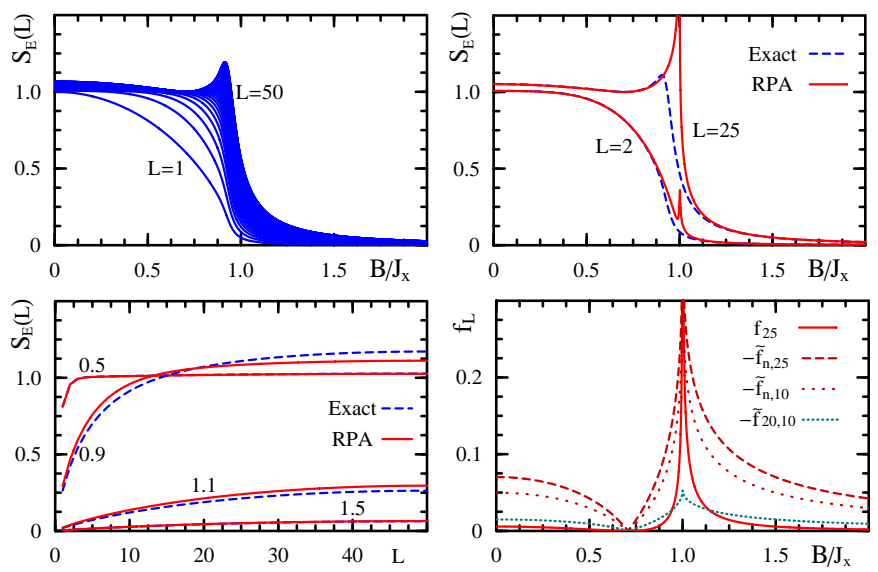

FIG. 3. Results for the fully connected spin $1 / 2$ array of $n=100$ spins. Top: Left: Exact entanglement entropies $S_{E}(L)=S\left(\rho_{L}\right)$ of subsystems with $L \leq n / 2$ spins as a function of the magnetic field. Right: Comparison between exact and RPA results for $S\left(\rho_{L}\right)$. Bottom: Left: Exact and RPA results for $S\left(\rho_{L}\right)$ as a function of the subsystem size $L$ at four different field ratios $B / B_{c}$. Right: Magnetic behavior of the average boson occupation number (67) for $L=25$ and the negative symplectic eigenvalue (70) of the partial transposed contraction matrix for different $L, m$.

where $\delta=0(1)$ for $|B|<B_{c}\left(\left(B / B_{c}\right)^{2 L s} \ll 1\right)$ and

$$
\Delta=\frac{n^{2}\left(\lambda^{2}-\bar{\omega}^{2}\right)}{2(n-1) \omega_{0} \omega_{1}}, \quad \bar{\omega}=\frac{\omega_{0}+(n-1) \omega_{1}}{n} .
$$

For $n=2$ we recover Eqs. (61) -62), while for large $n$, $\Delta \approx \frac{\lambda-\Delta_{+}^{0}}{\omega_{0}}-1$. Entanglement is then driven again by the ratio $\frac{\lambda^{2}-\bar{\omega}^{2}}{\omega_{0} \omega_{1}}$, which is small away from $B_{c}$ and vanishes at $B_{s}$. For small $\Delta, f_{L} \approx \frac{1}{2} \alpha_{L} \Delta$, with $\Delta \approx \frac{1}{2}\left(\frac{n}{(n-1)} \frac{J_{x}-J_{y}}{2 B}\right)^{2}$ for $|B| \gg B_{c}$ and $\Delta \propto\left(B-B_{s}\right)^{2}$ in the vicinity of $B_{s}$. For $B \rightarrow B_{c}, f_{L} \propto \sqrt{\alpha_{L}}\left(B-B_{c}\right)^{-1 / 4}$ and $S\left(\rho_{L}\right) \approx \log _{2} f_{L} e$.

The bosonic negativity of a bipartition $(m, L-m)$ of a subsystem of $L \leq n$ spins can again be explicitly obtained, since there is also a single negative eigenvalue $\tilde{f}_{L m}$ of the partial transpose of the contraction matrix (see appendix B):

$$
\begin{aligned}
N_{m, L-m} & =\frac{-\tilde{f}_{L m}}{1+2 \tilde{f}_{L m}}, \\
\tilde{f}_{L m} & =\frac{1}{2} \sqrt{1+\gamma_{L m} \Delta-\sqrt{8 \beta_{L m} \Delta+\gamma_{L m}^{2} \Delta^{2}}}- \\
\gamma_{L m} & =\alpha_{L}+4 \beta_{L m}, \quad \beta_{L m}=m(L-m) / n^{2}
\end{aligned}
$$

For a global partition $(L=n), \alpha_{n}=0$ while $\beta_{n m}=$ $\alpha_{m}$, and $\tilde{f}_{n L}=f_{L}-\sqrt{f_{L}\left(f_{L}+1\right)}$, with $N_{n L}=f_{L}+$ $\sqrt{f_{L}\left(f_{L}+1\right)}$, as in Eq. (63). In general, for small $\Delta$,

$$
\tilde{f}_{L m} \approx-\sqrt{\frac{1}{2} \beta_{L m} \Delta} \approx-\sqrt{\left(\beta_{L m} / \alpha_{L}\right) f_{L}}
$$

such that for strong fields, $\tilde{f}_{L m} \approx \sqrt{\beta_{L m}} \frac{n}{n-1} \frac{J_{x}-J_{y}}{4 B}$, while for $B$ close to $B_{s}, \tilde{f}_{L m} \propto \sqrt{\beta_{L m}}\left|B-B_{s}\right|$. On the other 


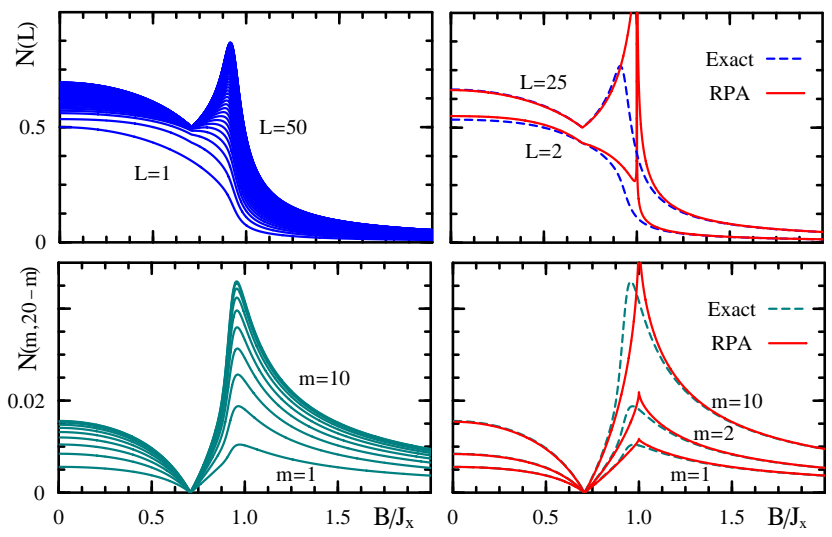

FIG. 4. Top: Left: Exact global negativities $N(L)=N_{L, n-L}$ between $L$ and $n-L$ spins in the fully connected array. Right: Comparison between exact and RPA results for $N(L)$ for two values of $L$. Bottom: Left: Exact subsystem negativities $N_{m, L-m}$ between $m$ and $L-m$ spins in a subsystem of $L=20$ spins. Right: Comparison between exact and RPA results for $N_{m, L-m}$.

hand, for $B \rightarrow B_{c}, \tilde{f}_{L m} \rightarrow-\frac{1}{2}\left(1-\sqrt{\frac{\alpha_{l}}{\alpha_{L}+4 \beta_{L m}}}\right)+O(\mid B-$ $\left.\left.B_{c}\right|^{1 / 2}\right)$ if $\alpha_{L} \neq 0$, implying that subsystem negativities $N_{m, L-m}$ with $L<n$ remain finite at $B_{c}$ (in agreement with the results of [16]), as $\tilde{f}_{L m}$ remains above $-\frac{1}{2}$.

In the parity breaking phase, the replacement (56) $\left(N \rightarrow 2 N+\frac{1}{2}\right)$ should be used for global negativities $N_{n, L-n}$, whereas subsystem negativities $N_{m, L-m}$ remain unchanged after parity restoration if $\left(B / B_{c}\right)^{2 s(n-L)}$ and $\left(B / B_{c}\right)^{2 s L}$ can both be neglected.

Eqs. (66)- (70) represent essentially the exact expressions for the subsystem entropy and negativity for large spin at finite $n$, as well as for large $n$ at finite spin, as verified by exact numerical calculations. For instance, exact (obtained through diagonalization of $H$ ) and RPA results for a spin $1 / 2$ array of $n=100$ spins are shown in figs. 3 4. RPA results for the entanglement entropy are quite accurate except in the vicinity of $B_{c}$, differences decreasing as $n$ or $s$ increases. For large $L$ they were obtained with the previous expression (66) whereas for small $L$ (like the $L=2$ case), we have used the proper spin state (54), whose main effect is to take into account the correct overlap for $B$ below but close to $B_{c}$ (roughly, $\delta$ replaced by the entropy of the reduced mean field superposition).

The variation of $S\left(\rho_{L}\right)$ with $L$ at fixed field (bottom left panel in Fig. 3) is also correctly predicted, being quite accurate both in the normal and parity breaking phase for fields not too close to $B_{c}$. The bottom right panel shows that $f_{L}$ remains small except for $B$ around $B_{c}$, while $\tilde{f}_{L m}$ becomes also small as $L$ decreases, in full agreement with Eq. (72). RPA results for global $\left(N_{n, L-n}\right)$ and in particular subsystem negativities $\left(N_{m, L-m}\right.$ for $\left.L<n\right)$, which are much smaller and vanish at $B_{s}$, are also very accurate, as seen in Fig. 4. Subsystem negativities were directly obtained with Eq. (69), whereas global negativities were corrected with Eq. (56) for $B<B_{c}$ and large $L$ and using (54) for $L=2$.

\section{DISCUSSION}

We have shown that the mean field plus RPA method is able to provide, through the bosonic representation, a general tractable method for estimating, in the ground state of general spin arrays, the entanglement entropy of any bipartition of the whole system as well as the negativity associated with any bipartition of any subsystem. The approach becomes fully analytic in systems with translational invariance, where no numerical diagonalization is required for obtaining the basic contraction matrices.

The bosonic treatment provides essentially the exact behavior of the system in the large spin limit. Finite spin corrections can be taken into account through the corresponding RPA spin state, which allows in particular to implement the non-negligible symmetry restoration effects in the case of the parity-breaking mean field, but which otherwise yields result which are in full agreement with the bosonic treatment at first order in the average local boson occupation. The latter is normally very low away from critical regions.

Through direct application of the present method, simple analytic expressions for the entanglement entropy and negativities for a spin $s$ pair and for a fully connected array of $n$ spins $s$ in a uniform field, have been straightforwardly obtained, which depend explicitly on the RPA energies. The agreement with exact numerical results is confirmed to improve as the spin $s$ increases at fixed size, and in the fully connected case also as $n$ increases at fixed $s$, differences being in fact negligible away from the critical region for not too small $s$ or size.

An important general prediction that arises from the present treatment is that entanglement from elementary excitations approaches a non-vanishing spin independent limit as the spin increases. An RPA quantum regime, characterized by weak entanglement, emerges then between strictly classical and strongly quantum regimes.

The authors acknowledge support from CIC (RR) and CONICET (JMM,NC) of Argentina.

\section{Appendix A: RPA spin densities}

We will here construct the spin density matrices compatible with the RPA spin state (14) and the contractions (17) up to second order in $V$, i.e., first order in the average occupation $V V^{\dagger}$ (implying zero or one boson per site). At this order, $F \approx G G^{\dagger}$ (Eqs. (17)) and the support of $\rho=\left|0_{\mathrm{RPA}}\right\rangle\left\langle 0_{\mathrm{RPA}}\right|$ is just the subspace spanned by the mean field state $|0\rangle$ plus the two site excitations $\left|1_{i} 1_{j}\right\rangle$ (Eq. (15)), leading to

$$
\rho \approx\left(\begin{array}{cc}
\boldsymbol{G}^{\dagger} & \boldsymbol{G} \\
\boldsymbol{G}^{\dagger} & 1-\boldsymbol{G}^{\dagger} \boldsymbol{G}
\end{array}\right)
$$


where $\boldsymbol{G}$ denotes a column matrix of elements $G_{i j}, i<$ $j$. At this order, $\rho^{2}=\rho$. The ensuing reduced density matrix $\rho_{A}=\operatorname{Tr}_{\bar{A}} \rho$ of a subsystem $A$ of $L$ spins becomes

$$
\rho_{A} \approx\left(\begin{array}{ccc}
\boldsymbol{G}_{A} \boldsymbol{G}_{A}^{\dagger} & 0 & \boldsymbol{G}_{A} \\
0 & F_{A}-G_{A} G_{A}^{\dagger} & 0 \\
\boldsymbol{G}_{A}^{\dagger} & 0 & 1-\operatorname{tr} F_{A}+\boldsymbol{G}_{A}^{\dagger} \boldsymbol{G}_{A}
\end{array}\right)\left(\mathrm{A}_{2}\right)
$$

where $F_{A}, G_{A}$ are the $L \times L$ contraction matrices of subsystem $A$ and $\boldsymbol{G}_{A}$ the concomitant column vector (of length $L(L-1) / 2$ ). The central block contains the one-site elements $|1 i\rangle\left\langle 1_{j}\right|$ arising from the partial trace of $\boldsymbol{G} \boldsymbol{G}^{\dagger}$. Here we have used the approximate identity $\sum_{k \in \bar{A}} G_{i k} G_{k j}^{\dagger} \approx F_{i j}-\sum_{k \in A} G_{i k}^{\dagger} G_{j k}$ for $i, j \in A$ (and neglected diagonal elements $G_{i i}$, of higher order due to the absence of self-energy terms), which allows to write $\rho_{A}$ entirely in terms of local contractions. Eq. (A2) is then in agreement with direct state tomography at this order (for $i, j, k, l \in A,\left\langle b_{j}^{\dagger} b_{i} \prod_{k \neq i, j}\left(1-b_{k}^{\dagger} b_{k}\right)\right\rangle_{0^{\prime}} \approx\left(F_{A}-G_{A} G_{A}^{\dagger}\right)_{i j}$, $\left.\left\langle b_{i}^{\dagger} b_{j}^{\dagger} b_{k} b_{l}\right\rangle_{0^{\prime}} \approx G_{k l} \bar{G}_{i j}\right)$. Up to $O\left(V^{2}\right), \rho_{A}$ is a positive matrix with $\operatorname{Tr} \rho_{A}=1$, but is no longer pure.

Its entropy $S\left(\rho_{A}\right)=-\operatorname{Tr} \rho_{A} \log _{2} \rho_{A}$ is determined, at this order, by the central block $\rho_{A}^{1}=F_{A}-G_{A} G_{A}^{\dagger}$,

$$
S\left(\rho_{A}\right) \approx \operatorname{tr} \rho_{A}^{1}\left(\log _{2} e-\log _{2} \rho_{A}^{1}\right),
$$

which coincides with Eq. (25) up to second order in $V$ (at this order $f_{A}^{\alpha}$ coincides with the eigenvalues of $\rho_{A}^{1}$ and Eq. (25) becomes $\left.\approx \sum_{\alpha} f_{A}^{\alpha}\left(\log _{2} e-\log _{2} f_{A}^{\alpha}\right)\right)$.

On the other hand, the leading term in the negativity arising from a bipartition $(B, C)$ of $A$ is of first order in $V$ and is just the sum of the singular values of the submatrix $G_{B C}$ (of elements $G_{i j}, i \in B, j \in C$ ), whence $N_{B C} \approx \operatorname{tr}\left[G_{B C} G_{B C}^{\dagger}\right]^{1 / 2}$. At this order, the negative symplectic eigenvalues $\tilde{f}_{A}^{\alpha}$ in (29) are again minus the singular values of $G_{B C}$, while Eq. (28) becomes $N_{B C} \approx-\sum_{\alpha} \tilde{f}_{A}^{\bar{\alpha}}$, leading again to the previous result.

Let us finally notice that Eq. (A2) always commutes with the $S_{z}$ parity (along the mean field axis) of subsystem $A$, i.e., $\left[\rho_{A}, P_{z A}\right]=0, P_{z A}=\exp \left[i \pi \sum_{i \in A}\left(s_{i z}-s_{i}\right)\right]$. In the case of two spins $i, j, \boldsymbol{G}_{A}$ has length 1 and Eq. (A2) is just a $4 \times 4$ blocked matrix, while in the case of a single spin $i, \boldsymbol{G}_{A}$ has length 0 and Eq. A2 becomes just $\rho_{i} \approx F_{i i}\left|1_{i}\right\rangle\left\langle 1_{i}\left|+\left(1-F_{i i}\right)\right| 0_{i}\right\rangle\left\langle 0_{i}\right|$.

\section{Appendix B: Fully connected system}

In the fully connected $X Y Z$ spin system, the contractions (65) are of the form $F_{i j}=F_{0} \delta_{i j}+F_{1}, G_{i j}=$ $G_{0} \delta_{i j}+G_{1}$, with $F_{0}, F_{1}, G_{0}, G_{1}$ real. The ensuing contraction matrix $\mathcal{D}_{L}$ for a subsystem of $L$ spins will then have symplectic eigenvalues (see also [26])

$$
\begin{aligned}
& f_{L}=\sqrt{\left(F_{0}+L F_{1}+\frac{1}{2}\right)^{2}-\left(G_{0}+L G_{1}\right)^{2}}-\frac{1}{2} \\
& f_{0}=\sqrt{\left(F_{0}+\frac{1}{2}\right)^{2}-G_{0}^{2}}-\frac{1}{2}
\end{aligned}
$$

plus their partners $1+f_{L}, 1+f_{0}$, where $f_{L}$ is nondegenerate while $f_{0}$ has $L-1$ degeneracy. Eqs. (B1) (B2) can be obtained either by a Fourier transform of the local operators or by noticing that the $L \times L$ contraction matrix $F_{L}$ can be written as $F_{L}=F_{0} I_{L}+F_{1} \mathbf{1}_{L} \mathbf{1}_{L}^{t}$ (and similarly for $G_{L}$ ), with $I_{L}$ the $L \times L$ identity and $\mathbf{1}_{L}$ a column $L \times 1$ vector with unit elements. $F_{L}$ and $G_{L}$ will then be diagonal in the same local basis with eigenvalues $F_{0}+L F_{1}$ and $F_{0}(L-1$ degenerate $)$ and similarly for $G_{L}$, which leads to Eqs. (B1)-(B2). In the case of a global vacuum, $f_{0}=0$ (since for $L=n$, we should have $f_{L=n}=f_{0}=0$ ), implying a single positive eigenvalue $f_{L}$ for any $L<n$. Eq. (B1) leads then to Eq. (67).

For evaluating the negativity $N_{m p}$ of a bipartition $(m, p)$ of a subsystem of $L$ spins $(m+p=L)$, we may first note that $F_{L}$ will be composed of blocks $F_{m m}=F_{0} I_{m}+F_{1} \mathbf{1}_{m} \mathbf{1}_{m}^{t}, F_{m p}=F_{1} \mathbf{1}_{m} \mathbf{1}_{p}^{t}=F_{p m}^{t}$ and $F_{p p}=F_{0} I_{p}+F_{1} \mathbf{1}_{p} \mathbf{1}_{p}^{t}$, and similarly for $G_{L}$. A local transformation allows then to write $F_{L}$ as a direct sum of a $(L-2) \times(L-2)$ diagonal block $F_{0} I_{L-2}$ plus the block $F_{0} I_{2}+F_{1}\left(\begin{array}{c}m \sqrt{m p} \\ \sqrt{m p} p\end{array}\right)$, and similarly for $G_{L}$. The ensuing partially transposed contraction matrix will then have symplectic eigenvalues $\tilde{f}_{0}=f_{0}($ Eq. $(\bar{B} 2)), L-2$ degenerate (with $\tilde{f}_{0}=0$ for a global vacuum) and

$$
\tilde{f}_{L m}^{ \pm}=\frac{1}{2} \sqrt{\operatorname{Tr} \mathcal{A}^{2} \pm \sqrt{\left(\operatorname{Tr} \mathcal{A}^{2}\right)^{2}-16 \operatorname{det} \mathcal{A}}}-\frac{1}{2}
$$

together with their partners $1+\tilde{f}_{0}, 1+\tilde{f}_{L m}^{ \pm}$, where $\mathcal{A}=\left(\begin{array}{c}A_{F G}-A_{G F} \\ A_{G F}-A_{F G}\end{array}\right)$ is a $4 \times 4$ matrix with blocks $A_{F G}=$ $\left(\frac{1}{2}+F_{0}\right) I_{2}+\left(\begin{array}{cc}m F_{1} & \sqrt{m p} G_{1} \\ \sqrt{m p} G_{1} & p F_{1}\end{array}\right)$ and similarly for $\mathcal{A}_{G F}$. Here $\tilde{f}_{L M}^{+}>0$ but $\tilde{f}_{L M}^{-}<0$. The latter is the single negative symplectic eigenvalue given in Eq. (70).
[1] M.A. Nielsen and I. Chuang, Quantum Computation and Quantum Information, Cambridge Univ. Press (2000).

[2] L. Amico, R. Fazio, A. Osterloh and V. Vedral, Rev. Mod. Phys. 80, 516 (2008).

[3] J. Eisert, M. Cramer, M.B. Plenio, Rev. Mod. Phys. 82, 277 (2010).

[4] T.J. Osborne, M.A. Nielsen, Phys. Rev. A 66, 032110 (2002).

[5] G. Vidal, J.I. Latorre, E. Rico and A. Kitaev, Phys. Rev. Lett. 90, 227902 (2003).
[6] T. Kashiwa, Y. Ohnuki, and M. Susuki, Path Integral Methods, Oxford University Press, Oxford (1997).

[7] S.R. White, Phys. Rev. Lett. 69, 2863 (1992); U. Schollwck, Rev. Mod. Phys. 77, 259 (2003).

[8] F. Verstraete and J.I. Cirac, Phys. Rev. B 73, 094423 (2006).

[9] N. Canosa, J.M. Matera, and R. Rossignoli, Phys. Rev. A 76, 022310 (2007); J.M. Matera, R. Rossignoli, and N. Canosa, Phys. Rev. A 78012316 (2008).

[10] J.M. Matera, R. Rossignoli, and N. Canosa, Phys. Rev. 
A 78, 042319 (2008)

[11] L. Amico et al, Phys. Rev. A 74, 022322 (2006); F. Baroni et al, J. Phys. A 409845 (2007).

[12] R. Rossignoli, N. Canosa, and J.M. Matera, Phys. Rev. A 77, 052322 (2008).

[13] G. Vidal and R.F. Werner, Phys. Rev. A 65, 032314 (2002).

[14] K. Zyczkowski, P. Horodecki, A. Sanpera, and M. Lewenstein, Phys. Rev. A 58, 883 (1998); K. Zyczkowski, ibid. 60, 3496 (1999).

[15] H. Wichterich, J. Molina-Vilaplana, and S. Bose, Phys. Rev. A 80 010304(R) (2009).

[16] H. Wichterich, J. Vidal, and S. Bose Phys. Rev. A 81, 032311 (2010).

[17] N. Canosa and R. Rossignoli, P hys. Rev. A 73022347 (2006); R. Rossignoli and N. Canosa, Phys. Rev. A 72 012335 (2005).

[18] Peter Ring and Peter Schuck, The Nuclear Many-Body Problem, Springer-Verlag (1980).

[19] T. Holstein and H. Primakoff, Phys. Rev. 58, 1098 (1940).

[20] A. Klein and E.R. Marshalek, Rev. Mod. Phys. 63, 375 (1991).
[21] J. Vidal, S. Dusuel, and T. Barthel, J. Stat. Mech. (2007) P01015

[22] J. Kurmann, H. Thomas, and G. Müller, Physica A 112, 235 (1982).

[23] S.M. Giampaolo, G. Adesso, and F. Illuminati, Phys. Rev. Lett. 100, 197201 (2008); Phys. Rev. B 79, 224434 (2009).

[24] R. Rossignoli, N. Canosa, and J.M. Matera, Phys. Rev. A 80, 062325 (2009).

[25] K. Audenaert, J. Eisert, M.B. Plenio, R.F. Werner, Phys. Rev. A 66042327 (2002); M. Cramer, J. Eisert, M.B. Plenio, J. Dreißig, Phys. Rev. A 73012309 (2006).

[26] G. Adesso, A. Serafini, and F. Illuminati, Phys. Rev. A 70, 022318 (2004); A. Serafini, G. Adesso, and F. Illuminati, Phys. Rev. A 71, 032349 (2005); G. Adesso and F. Illuminati, Phys. Rev. A 78, 042310 (2008).

[27] H.J. Lipkin, N. Meshkov, and A.J. Glick, Nucl. Phys. 62, 188 (1965).

[28] J.I. Latorre, R. Orús, E. Rico, J. Vidal, Phys. Rev. A 71, 064101 (2005).

[29] T. Barthel, S. Dusuel, and J. Vidal, Phys. Rev. Lett. 97, 220402 (2006).

[30] S. Dusuel and J. Vidal, Phys. Rev. B 71, 224420 (2005). 